Research Paper

\title{
Modeling Efficacy of Bevacizumab Treatment for Meta- static Colon Cancer
}

\author{
Rezwan Islam ${ }^{1}$, Po-Huang Chyou ${ }^{2}$, and James K Burmester ${ }^{3}$ \\ 1. Department of Oncology/Hematology, Marshfield Clinic Weston Center, Marshfield, WI, USA; \\ 2. Biomedical Informatics Research Center, Marshfield Clinic Research Foundation, Marshfield, WI, USA; \\ 3. Clinical Research Center, Marshfield Clinic Research Foundation, Marshfield, WI, USA.
}

$\square$ Corresponding author: Rezwan Islam, MD. Marshfield Clinic Weston Center, Department of Oncology/Hematology, 3501 Cranberry Blvd, Weston, WI 54476. Phone: 715-393-1400 Fax: 715-389-5757 Email: islam.rezwan@marshfieldclinic.org.

() Ivyspring International Publisher. This is an open-access article distributed under the terms of the Creative Commons License (http://creativecommons.org/ licenses/by-nc-nd/3.0/). Reproduction is permitted for personal, noncommercial use, provided that the article is in whole, unmodified, and properly cited.

Received: 2013.02.16; Accepted: 2013.04.23; Published: 2013.04.29

\begin{abstract}
Purpose: Bevacizumab, an FDA-approved adjuvant treatment for metastatic colon cancer, has extended survival for many patients. However, factors predicting response to treatment remain undefined.

Patients and Methods: Relevant clinical and environmental data were abstracted from medical records of 149 evaluable patients treated with bevacizumab for metastatic colon cancer at a multi-specialty clinic. Tumor response was calculated from radiologic reports using Response Evaluation Criteria in Solid Tumors (RECIST) criteria and verified by oncologist review. Patients with at least one occurrence of complete or partial response or stable disease were classified as responders; those exhibiting progressive disease were classified as non-responders.

Results: Univariate analysis demonstrated that blood in stool $(P<0.05)$, unexplained weight loss $(P<0.05)$, primary colon cancer site $(P<0.05)$, chemotherapy treatment of primary tumor site $(P<0.05)$, and adenocarcinoma versus adenoma subtype $(P<0.05)$ was associated with tumor responsiveness. Factors remaining statistically significant following multivariate modeling included adenocarcinoma as tumor cell type versus other adenocarcinoma subtypes

$(\mathrm{OR}=6.35,95 \% \mathrm{Cl}$ : I.08-37.18), chemotherapy treatment applied to primary tumor $(\mathrm{OR}=0.07$, $95 \% \mathrm{Cl}: 0.0-0.76$,), tumor localization to cecal/ascending colon (OR=0.06I, $95 \% \mathrm{Cl}: 0.006-0.588$,), and unexplained weight loss (OR=0.I, $95 \% \mathrm{Cl}: 0.02-0.56$,). Chemotherapy treatment of primary tumor, unexplained weight loss, and cecal/ascending localization of the tumor were associated with poorer outcomes. Adenocarcinoma as cell type compared to other adenocarcinoma subtypes was associated with better response to bevacizumab treatment.

Conclusion: Results suggest that response to bevacizumab therapy may be predicted by modeling clinical factors including symptomology on presentation, tumor location and type, and initial response to chemotherapy.
\end{abstract}

Key words: Bevacizumab; Colon cancer; Monoclonal antibodies; Prognosis; Vascular endothelial growth factor/VEGF.

\section{INTRODUCTION}

Colon cancer is the third most common cancer diagnosed in the United States (excluding skin cancer), with about 101,000 new cases diagnosed during
2011. ${ }^{1}$ Colon cancer is the second leading cause of cancer death in the US with about 50,000 deaths occurring annually. ${ }^{1}$ 
In colon cancer increased expression of vascular endothelial growth factor (VEGF) occurs concurrently with increasing vascular density of an invasive tumor in the context of metastasis, and recurrence. Bevacizumab is a recombinant monoclonal antibody that specifically blocks the activity of human VEGF. ${ }^{2}$ VEGF is a potent, specific stimulator of angiogenesis supporting vascularization of growing tumors. ${ }^{3}$ In the absence of VEGF tumors become hypoxic. Bevacizumab treatment causes prolonged hypoxia resulting in vascular cell death primarily through apoptosis and decreased blood supply to the tumor. ${ }^{4}$

Clinical trials demonstrated an increase in median survival for colon cancer patients when treated with bevacizumab as an adjunct to standard chemotherapy. ${ }^{5-8}$ The addition of intravenous bevacizumab to bolus fluorouracil/folinic acid (FU/FA), irinotecan/bolus fluorouracil/folinic acid (IFL), irinotecan/infusional fluorouracil/folinic acid (FOLFIRI), infusional fluorouracil/folinic acid/oxaliplatin (FOLFOX), capecitabine/oxaliplatin (XELOX), or bolus fluorouracil/folinic acid/oxaliplatin has shown efficacy as a first-line therapy for metastatic colorectal cancer. In most randomized controlled trials, the addition of bevacizumab to standard treatment increased median overall survival by about 5 months for first-line therapy. ${ }^{5-7}$ The increase in survival in second-line therapy was about 2 months. ${ }^{8}$

Adverse drug events (ADE) such as gastrointestinal perforation, wound healing complications, and hemorrhage are increased in patients treated with bevacizumab. ${ }^{9}$ Further, the cost of bevacizumab is very high due to the extensive effort required to produce an injectable product. ${ }^{10}$ Thus, methods are needed to characterize which patients will derive the greatest benefit from bevacizumab treatment and distinguish them from non-responders and/or patients at ADE risk following exposure.

To investigate personal and medical factors that may influence which patients will have a positive response to bevacizumab, we collected data on 149 patients treated with bevacizumab for metastatic colon cancer.

\section{PATIENTS AND METHODS}

\section{Subject Recruitment}

The study was approved by the Marshfield Clinic Research Foundation's Institutional Review Board. An electronic search of the Marshfield Clinic electronic medical record identified 229 patients who had been treated with bevacizumab as part of first-line or second-line chemotherapy for metastatic colon cancer since 2002. Further screening of subjects to confirm eligibility for inclusion in the study based on inclusion criteria (metastatic colon cancer treated with bevacizumab) and verification of sufficient evaluable data (radiological data, and physician notes) determined 149/229 electronic met criteria for inclusion in the analysis.

\section{Data Abstraction}

Data abstracted from the medical records of patients validated as meeting inclusion criteria following screening $(n=149)$ included: height and weight (to determine body mass index), primary site of colon cancer, co-morbidities (including other cancers, heart disease, cardiovascular disease, diabetes mellitus, Crohn's disease, diverticulitis, and irritable bowel syndrome), unexplained or severe weight loss (defined as loss of $10 \%$ or greater of body weight), current age, age at colon cancer diagnosis, family history of colon cancer, stage and grade of primary tumor, number, site, stage, and grade of metastases, imaging scan results, treatment of the primary tumor including surgery and/or chemotherapy, and/or radiation, treatment of the primary metastasis including surgery, and/or chemotherapy, and/or radiation, dates and doses of chemotherapy, dates and doses of bevacizumab therapy, and all ADE (gastrointestinal perforation, wound healing complications, hemorrhage). Complete data were not consistently available for all 229 patients initially identified by electronic screening for all variables since each patient presented as an individual case and only patients who met inclusion criteria and had sufficient evaluable data were included in the analysis $(n=149)$. Tumor response was calculated from radiologic images using Response Evaluation Criteria in Solid Tumors (RECIST) guidelines ${ }^{11}$ for evaluating tumor response in oncology trials and compared to oncologists' assessment captured in the patients' medical records. Records were systematically checked following each bevacizumab treatment to determine if new radiographs had been taken and were available for RECIST evaluation. Response criteria were classified in compliance with RECIST categorization as follows: complete response (disappearance of all lesions), partial response (30\% decrease of tumor dimension from baseline measures), stable disease (neither response nor progression), and progressive disease (20\% increase). Patients were categorized as responders if they exhibited complete or partial response or exhibited stable disease at least once following response to any treatment that included bevacizumab administration. For analytical purposes, complete response, partial response, and stable disease were grouped together as responders reflecting disease control, while patients 
exhibiting progressive disease were grouped as non-responders (poorer outcome). ${ }^{12}$

\section{Statistical Analysis}

Colon cancer patients were divided into two groups based on the positive response status (yes/no) to bevacizumab. In the univariate analysis, the descriptive statistics (for any continuous measurements: mean, standard deviation, median, and the range; for any categorical measurements: frequency and percentage) were reported for each of the patient's attributes such as body mass index, age at diagnosis, family history of colon cancer, and other clinical characteristics. These risk factors were compared between the two groups using the t-test for normally distributed data, the Wilcoxon rank sum test for skewed data (the adverse event count), and the Fisher's exact test for cells with a value $<5$ or the Chi-square test for categorical data. Odds ratio (OR) and corresponding 95\% confidence interval (CI) for the status of positive response to bevacizumab treatment were derived from unconditional logistic regression modeling approach using each of the above-named risk factors as the predictor variable. A $P$-value of $<0.05$ was considered statistically significant. In multivariate analysis, a stepwise (forward selection and backward elimination) logistic regression modeling was performed to determine the set of important predictors, using the status of positive response to bevacizumab treatment as the dependent variable and risk factors that showed a significant $P$-value in the univariate analysis as the independent variables. All statistical analyses were carried out using commercially available statistical software package (SAS).

\section{RESULTS}

A total of 229 patients who had been treated with bevacizumab for metastatic colon cancer were evaluated for inclusion in the study. Of these, 149 met inclusion criteria, and had sufficient evaluable data available to qualify them for inclusion in the analyses (see Figure 1.) Table 1 summarizes demographic data for subjects grouped in accordance with response status of the subjects (i.e., responder, defined as having as least one full or partial response characterized as $>30 \%$ reduction in tumor size from baseline or stable disease in response to bevacizumab treatment, versus non-responder). No statistically significant differences between individuals meeting criteria as responders and non-responders were observed for racial background, ethnic background, gender, or smoking status. At the time of enrollment into the study, 135/149 patients were living and provided informed consent, and 14/149 patients were deceased.

Patients Identified Electronically as Having Been Treated with Bevacizumab: $(n=229)$

Records Not Abstracted ( $n=28)$,

due to:

- clinical outcome or status was uninterruptable $(n=3)$

- no metastatic disease confirmed or metastases removed $(n=7)$

- insufficient clinical/radiographic data available $(n=4)$

- treatment with bevacizumab not confirmable $(n=2)$

- did not meet inclusion criteria $(n=12)$, due to:

- mixture of cancers or wrong organ $(n=6)$

- complete response $(n=2)$

○ progression $(n=1)$

○ wrong stage $(n=3)$

Excluded because no informed consent attainable $(n=28)$ due to:

- cognitive issues $(n=3)$

- enrollment in hospice/ palliative care $(n=4)$

- declined enrollment $(n=6)$

- participation in other trial precluding enrollment in other studies $(n=2)$

- insufficient data capture due to nursing home residency or not an established Clinic patient $(n=13)$

Total number included in study: $n=149$

Fig I. Flow diagram of study enrollment. 
Table I. Positive tumor response related to patient demographics.

\begin{tabular}{llllll}
\hline \multicolumn{6}{l}{ Positive Tumor Response } \\
\hline & No & \multicolumn{5}{l}{ Yes } & \\
\hline Characteristics & No. & $\mathbf{( \% )}$ & No. & $\mathbf{( \% )}$ & P-value $^{*}$ \\
\hline Racial background & & & & &. $\mathbf{1 0 4 4}$ \\
$\quad$ Asian & 1 & $(7)$ & 0 & $(0)$ & \\
White or Caucasian & 13 & $(93)$ & 129 & $(96)$ & \\
Other & 0 & $(0)$ & 6 & $(4)$ & \\
Ethnic Background & & & & & $\mathbf{1 . 0 0 0 0}$ \\
Hispanic or Latino & 0 & $(0)$ & 3 & $(2)$ & \\
Not Hispanic or Latino & 11 & $(100)$ & 119 & $(98)$ & \\
Gender & & & & & .2657 \\
Male & 5 & $(36)$ & 72 & $(53)$ & \\
Female & 9 & $(64)$ & 63 & $(47)$ & \\
Smoking Status & & & & & .6448 \\
Current & 1 & $(8)$ & 27 & $(20)$ & \\
Ex-Smoker & 6 & $(46)$ & 56 & $(42)$ & \\
Never Smoker & 6 & $(46)$ & 49 & $(37)$ & \\
\hline
\end{tabular}

${ }^{*}$ P-value was derived from Fisher's Exact Test.

To determine whether existing medical conditions, family history of cancer, treatment for colon cancer (surgery, radiation, or chemotherapy), and adverse drug responses are associated with a positive tumor response, we collected this information from the patients' medical records in sequential fashion corresponding to each drug treatment. No differences between responders and non-responders were noted for any other factors evaluated including symptomology, comorbidities, tumor characteristics, or genetic factors, with the exception of those shown in Table 2. Significant differences between responders and non-responders detected in univariate analysis included blood in the stool $(P<0.05)$, unexplained weight loss $(P<0.05)$, primary site of colon cancer (cecal/ascending) $(P<0.05)$, adenocarcinoma type vs. all other adenocarcinoma subtypes $(P<0.05)$, and chemotherapy treatment of the primary tumor $(P<0.05)$ (see Table 2). Blood in the stool correlated with response in the subset of patients with adenocarcinoma as tumor type $(P<0.05)$. No statistical difference was noted between responders and non-responders for other treatment approaches to primary tumor including radiation therapy or surgery.

Multivariate stepwise logistic regression analysis was next applied to determine the set of important predictors for positive response to bevacizumab treatment. Among the variables modeled by multivariate analysis were those that had achieved statistical significance by univariate analysis with the exception of blood in stool. Inclusion of blood in stool was precluded because no patients among the non-responders exhibited this characteristic and thus comparison was not possible. Blood in stool was defined as observation of blood in stool by the patient, and in many cases, this was the symptom that brought the patient to the clinic for further evaluation. Variables that remained significant following multivariate regression analysis included adenocarcinoma as tumor cell type compared to other adenocarcinoma subtypes (OR=6.35, 95\% CI:1.08-37.18;), chemotherapy treatment approach to the primary tumor (OR=0.07, 95\% CI: $0.0-0.76$,), tumor localization to cecal/ascending colon (OR=0.061, 95\% CI: 0.006-0.588), and unexplained weight loss $(\mathrm{OR}=0.1$, 95\% CI: 0.02-0.56,).

Table 2. Positive tumor response related to patient medical condition with statistical differences between responders and non-responders: univariate analysis.

\begin{tabular}{llllll}
\hline & \multicolumn{5}{l}{ Positive Tumor Response } \\
\hline & No & \multicolumn{5}{c}{ Yes } & \\
\hline Characteristics & No. & (\%) & No. & (\%) & P-value* $^{*}$ \\
\hline $\begin{array}{l}\text { Blood in the stool } \\
\text { Yes }\end{array}$ & 0 & $(0)$ & 62 & $(100)$ & \\
No & 11 & $(16)$ & 58 & $(84)$ & \\
$\begin{array}{l}\text { Unexplained weight loss } \\
\text { Yes }\end{array}$ & 7 & $(17)$ & 35 & $(83)$ & \\
No & 4 & $(4)$ & 85 & $(96)$ & \\
Cecal/ascending & & & & & .0072 \\
Yes & 10 & $(17)$ & 50 & $(83)$ & \\
No & 3 & $(3)$ & 85 & $(97)$ & \\
$\begin{array}{l}\text { Chemotherapy treatment of } \\
\text { primary tumor }\end{array}$ & & & & & .0456 \\
Yes & 12 & $(13)$ & 77 & $(87)$ & \\
No & 2 & $(3)$ & 58 & $(97)$ & \\
\hline
\end{tabular}

*P-value was derived from Fisher's Exact Test.

Table 3. Multivariate Stepwise Logistic Regression Analysis for Selected Predictor Clinical Characteristics in Association with Positive Tumor Response among Colon Cancer Patients.

\begin{tabular}{|c|c|c|c|}
\hline Selected clinical characteristics* & OR & CI§ & P-valueq \\
\hline \multicolumn{4}{|l|}{ Adenocarcinoma } \\
\hline Yes & 6.35 & $1.08-37.18$ & 0.03 \\
\hline No† & 1.00 & & \\
\hline \multicolumn{4}{|l|}{ Chemotherapy } \\
\hline Yes & 0.069 & $0.006-0.757$ & 0.0176 \\
\hline No† & 1.00 & & \\
\hline \multicolumn{4}{|l|}{$\begin{array}{l}\text { Tumor site: Cecal/ascending } \\
\text { colon }\end{array}$} \\
\hline Yes & 0.06 & & \\
\hline No† & 1.00 & $0.006-0.588$ & 0.0008 \\
\hline \multicolumn{4}{|l|}{ Unexplained weight loss } \\
\hline \multicolumn{4}{|l|}{ Yes } \\
\hline No† & 0.10 & $0.02-0.56$ & 0.006 \\
\hline
\end{tabular}




\section{DISCUSSION}

Efficacy of first-line/second-line bevacizumab treatment in combination with chemotherapy to extend progression-free survival and overall survival for many patients with metastatic colon cancer has been established.7,8 At the same time, an important subset of patients do not benefit from bevacizumab therapy. To date, few markers have been explored to distinguish potential responders from non-responders with respect to treatment with bevacizumab. Markers of response to bevacizumab that have previously been reported for metastatic colon cancer include carbonic anhydrase 9,13 visceral fat area, ${ }^{14}$ and hypertension. ${ }^{15}$ Studies also suggest that heritable genetic polymorphisms may contribute to bevacizumab responsiveness in breast cancer, since single nucleotide polymorphisms (SNPs) in VEGF were associated with better overall survival. ${ }^{16}$ It is unknown whether these same DNA variants are also predictive of response to bevacizumab in colon cancer patients. In contrast, tumor mutations in k-ras, b-raf, or p53 did not predict response to bevacizumab, 17 and tumor levels of VEGF, thrombospondin-2, and micro-vessel density did not predict response to bevacizumab. ${ }^{18}$

The high cost of bevacizumab treatment, ${ }^{10}$ coupled with substantial adverse drug events, ${ }^{9}$ makes it important to identify clinical markers that can be used to predict patient response to bevacizumab treatment. In this study, clinical variables that may be informative with respect to predicting response to treatment were explored. Variables that exhibited a statistically significant difference between responders (defined as having as least one full or partial response characterized as a $\geq 30 \%$ reduction in tumor size from baseline, or stable disease in response to bevacizumab treatment) versus non-responders included (1) cancer site (cecal/ascending colon; $37 \%$ of responders vs $77 \%$ of non-responders, compared to tumor localization in the transverse, descending/sigmoid or other site in the colon); (2) cancer cell type (adenocarcinoma, 87\% of responder vs. $62 \%$ non-responders, compared to adenocarcinoma subtypes including mucinous adenocarcinoma, adenocarcinoma/mucinous adenocarcinoma, adenocarcinoma/signet ring cell carcinoma, mucinous adenocarcinoma/signet ring cell carcinoma, adenocarcinoma/mucinous adenocarcinoma/signet ring cell carcinoma); (3) chemotherapy treatment of primary tumor $(57 \%$ of responders vs $86 \%$ of non-responders; (4) unexplained weight loss ( $29 \%$ of responders vs $64 \%$ of non-responders; and (5) blood in stool $(52 \%$ of responders and zero non-responders). $P$-values are shown in table 2 . When variables exhibiting statistically significant differences between responders and non-responders (excluding blood in stool due to lack of comparison group) were modeled in multivariate analysis, the following profile persisted relative to response to treatment: (1) patients with cecal/ascending colon site had worse outcomes (OR=0.06, CI=0.02-0.56,); (2) patients with adenocarcinoma versus other subtypes showed better response (OR=6.35, CI=1.08-37.18,); (3) patients initially treated with chemotherapy had worse response $(\mathrm{OR}=0.07, \mathrm{CI}=0.01-0.76$, ); and (4) patients who presented with unexplained weight loss had worse response to treatment $(\mathrm{OR}=0.10, \mathrm{CI}=0.02-0.56$,). These data suggest that differential response to bevacizumab may be due to inherent differences in tumor gene expression between adenocarcinoma cell type and other colon cancer cell types. The difference noted between primary cecal/ascending tumors and transverse/descending tumors may be due to both anatomical and gene expression differences inherent between ascending colon consistent with differences in function. The ascending colon actively resorbs water, whereas the descending colon is more actively involved in bacterially-mediated breakdown of nutrients and resorption of vitamins and trace minerals. Anatomical differences may also impact on drug resorption and distribution along the length of the colon and may contribute to lower efficacy of bevacizumab treatment observed in non-responders who were treated with chemotherapy as the initial approach to primary tumor treatment. Blood in the stool as the symptom that brought patients in for evaluation before diagnosis of colon cancer was also a strong marker of response to bevacizumab in univariate analysis. However, because we did not observe any non-responders with blood in the stool, this variable could not be modeled in the multivariate analysis and will require a larger trial to obtain an accurate estimate of its effect. This marker as a positive predictor of response to bevacizumab may be useful clinically, since it occurs early in the trajectory of colon cancer diagnosis and treatment. Similarly, unexplained sudden weight loss was more prevalent among non-responders and could be an important early clinical predictor of response to treatment.

A limitation of this study was the small number of non-responders identified. Thus, further studies to validate these findings and enhance modeling through addition of further factors predictive of patient response to bevacizumab treatment are warranted.

Statistically significant outcomes stemming from univariate and multivariate analysis and modeling of clinical data related to clinical factors suggest that profiling patients with respect to their clinical status 
may have important predictive value for anticipating response to bevacizumab treatment. In summary, multivariate analysis demonstrated that patients with pure adenocarcinoma are more likely to response to bevacizumab treatment compared to other types of tumors. Patients initially treated with chemotherapy treatment of the primary tumor are less likely to response compared to the patients who did not have chemotherapy. Patients who have unexplained weight loss are less likely to respond compared to patients without unexplained weight loss, and patients with cancer localized to the cecal/ascending colon were likely to have worse response to bevacizumab treatment. Univariate analysis suggested that patients presenting with blood in stool were more likely to respond to bevacizumab treatment, and no non-responders presented with blood in stool. Validation of these data in further studies is warranted since predictive value of these clinical observations may be important in informing treatment strategies of patients with colon cancer. This study underlines strong inherent value in advancing such modeling approaches in predicting patient response to treatment.

\section{ACKNOWLEDGEMENTS}

The authors thank Hilmi Ege, MD and Ingrid Glurich, Ph.D., for thoughtful discussion and suggestions during the project. The authors further thank Autumn Deedon for data collection, Terrie Kitchner and Kimberly Hill for medical record review and data quality assurance and Marie Fleisner for editorial assistance.

\section{Financial Support}

Funding for this study was provided by Disease Specific Funds from the Marshfield Clinic Research Foundation.

\section{Author Contributions}

Conception and design: All authors

Provision of study materials or patients: Rezwan Islam

Collection and assembly of data: Po-Huang Chyou and James K Burmester

Data analysis and interpretation: Po-Huang Chyou and James K Burmester

Manuscript writing: All authors

Final approval of manuscript: All authors

\section{CONFLICTS OF INTEREST}

All authors declare no financial conflicts of interest.

\section{REFERENCES}

1. Siegel R, Ward E, Brawley O, et al. Cancer statistics, 2011. CA Cancer J Clin, 2011; 61:212-236.

2. Shih T, Lindley C. Bevacizumab: an angiogenesis inhibitor for the treatment of solid malignancies. Clin Ther, 2006; 28:1779-1802.

3. Ferrara N, Davis-Smyth T. The biology of vascular endothelial growth factor. Endocr Rev 1997;18:4-25

4. Selvakumaran M, Yao KS, Feldman MD, et al. Antitumor effect of the angiogenesis inhibitor bevacizumab is dependent on susceptibility of tumors to hypoxia-induced apoptosis. Biochem Pharmacol 2008;75:627-638

5. Hurwitz H, Fehrenbacher L, Novotny W, et al. Bevacizumab plus irinotecan, fluorouracil, and leucovorin for metastatic colorectal cancer. $\mathrm{N}$ Eng J Med 2004;350:2335-2342

6. Hurwitz HI, Fehrenbacher L, Hainsworth JD, et al. Bevacizumab in combination with fluorouracil and leucovorin: an active regimen for first-line metastatic colorectal cancer. J Clin Onc 2005;23:3502-3508

7. McCormack PL, Keam SJ. Bevacizumab: a review of its use in metastatic colorectal cancer. Drugs 2008;68:487-506

8. Golfinopoulos V, Salanti G, Pavlidis N, et al. Survival and dis-ease-progression benefits with treatment regimens for advanced colorectal cancer: a meta-analysis. Lancet Oncol 2007;8:898-911

9. Higa GM, Abraham J. Biological mechanisms of bevacizumab-associated adverse events. Expert Rev Anticancer Ther 2009;9:999-1007

10. Tappenden P, Jones R, Paisley S, et al. The cost-effectiveness of bevacizumab in the first-line treatment of metastatic colorectal cancer in England and Wales. Eur J Cancer 2007;43:2487-2494

11. Therasse P, Arbuck SG, Eisenhauer EA, et al. New guidelines to evaluate the response to treatment in solid tumors. European Organization for Research and Treatment of Cancer, National Cancer Institute of the United States, National Cancer Institute of Canada. J Natl Cancer Inst 2000;92:205-216

12. Tuma RS. Sometimes size doesn't matter: reevaluating RECIST and tumor response rate endpoints. J Nat Cancer Inst 2006;98:1272-1274

13. Hong YS, Cho HJ, Kim SY, et al. Carbonic anhydrase 9 is a predictive marker of survival benefit from lower dose of bevacizumab in patients with previously treated metastatic colorectal cancer. BMC Cancer 2009;9:246-254

14. Guiu B, Petit JM, Bonnetain F, et al. Visceral fat area is an independent predictive biomarker of outcome after first-line bevacizumab based treatment in metastatic colorectal cancer. Gut 2010;59:341-347

15. Ryanne Wu R, Lindenberg PA, Slack R, et al. Evaluation of hypertension as a marker of bevacizumab efficacy. J Gastrointest Cancer, 2009; 40:101-108.

16. Schneider BP, Wang M, Radovich M, et al. Association of vascular endothelial growth factor and vascular endothelial growth factor receptor-2 genetic polymorphisms with outcome in a trial of paclitaxel compared with paclitaxel plus bevacizumab in advanced breast cancer: ECOG 2100. J Clin Oncol 2008;28:4672-4678

17. Ince WL, Jubb AM, Holden SN, et al. Association of k-ras, b-raf, and p53 status with the treatment effect of bevacizumab. J Natl Cancer Inst 2005;97:981-989

18. Jubb AM, Hurwitz HI, Bai W, et al. Impact of vascular endothelial growth factor-A expression, thrombospoindin-2 expression, and mi-cro-vessel density on the treatment effect of bevacizuamab in metastatic colorectal cancer. J Clin Oncol 2006;24:217-227 